\title{
CD68+ M1 MACROPHAGES IS ASSOCIATED WITH PLACENTAL INSUFFICIENCY UNDER FETAL GROWTH RESTRICTION
}

D01:10.36740/WLek202102107

\author{
Varvara A. Berezhna, Tetiana V. Mamontova, Antonina M. Gromova \\ UKRAINIAN MEDICAL STOMATOLOGICAL ACADEMY, POLTAVA, UKRAINE
}

\begin{abstract}
The aim: To elucidate the possible involvement of $\mathrm{M} 1$ and $\mathrm{M} 2$ macrophages in the placentas of women, whose pregnancies were complicated by fetal growth restriction (FGR) and resulted in term births after 37 weeks of gestation and preterm births up to 37 weeks of gestation.

Materials and methods: CD68+ and CD163+ macrophages were studied by immunohistochemical method, placental morphology in the placentas of 16 women whose pregnancies were complicated by FGR and resulted in term births at a gestational age after 37 weeks (1- ${ }^{\text {st }}$ group, $n=7$ ) or resulted in preterm births at a gestational age up to 37 weeks ( $2{ }^{\text {nd }}$ group, $n=9$ ). The control group consisted of 10 placentas of women with physiological pregnancies and births.

Results: Women 2-nd group showed significantly low weight of the placenta, a short gestation period at the time of delivery, and a prolonged labor period than women of the control group ( $p<0.001 ; p<0.001 ; p<0.05$, respectively). The level of CD68+ and CD163+ macrophages in the placentas of women 2- ${ }^{\text {nd }}$ group was significantly higher than in woman 1-st group ( $p<0.001, p<0.001$, respectively). A significant correlation was found between the expression level of CD68+ monocytes in the intervillous space and the weight of a newborn $(r=-0.765 ; p=0.016)$ in women $2^{\text {- }}{ }^{\text {d }}$ group.

Conclusions: These studies suggest that in the placentas of women whose pregnancies were complicated by FGR and resulted in preterm births, the increased activation of CD68+ macrophages of the pro-inflammatory pool may be associated with disorders of the vascular and stromal component of the villous chorion with the development of involutive and dystrophic changes. In general, this fact probably determines the progress of chronic placental insufficiency and aggravates the development of fetal growth restriction.
\end{abstract}

KEY WORDS: fetal growth restriction, preterm and in term birth, preterm and in term delivery, placental insufficiency, morphology of placenta, M1 and M2 macrophages

Wiad Lek. 2021;74(2):213-219

\section{INTRODUCTION}

Fetal growth restriction (FGR) is an urgent problem of modern medicine that occurs in 3-8\% of all pregnancies [1]. FGR is accompanied by an increasing risk of preterm birth, perinatal diseases and mortality, as well as an unfavorable course of adaptation processes in newborns [2]. In FGR, functional insufficiency of the placenta is observed, which is often registered during early termination of pregnancy in the form of preterm birth. Placental insufficiency is combined with structural and functional disorders, among which the inflammatory and immune processes are of particular importance [3]. Inflammation and activation of immune cells of the monocyte-macrophage system constitute an integral component of the major gestational events in physiological and pathological pregnancy, in particular under FGR.

Macrophages (Mops) play a key role in the immune defense and maintenance of homeostasis $[4,5]$. Monocyte-macrophage system is distinguished by a high degree of heterogenenicity and plasticity, depending on the microenvironmental factors. There are 2 types of Mops - M1 ("the classically activated type", pro-inflammatory) and M2 ("the alternatively activated type", anti-inflammatory). Mops in the placenta is associated with the involvement of these cells in the physiological and pathogenetic processes of pregnancy and childbirth at all stages. During physiological pregnancy, macrophages are balanced in the immunoregulatory phenotype and regulate the innate and adaptive immune response. It is obvious that Mops (decidual and Hofbauer cells) exhibit a predominantly anti-inflammatory $\mathrm{M} 2$ phenotype in the placenta. These Mops are involved in the tissue remodeling and homeostasis, in the maintaining the maternal and fetal tolerance, trophoblast invasion, spiral artery remodeling, as well as tissue regeneration and angiogenesis [6,7].

The data on macrophages in FGR are very limited. It was revealed that CD68+M1-macrophages have focal localization in the stroma of villi during pregnancy complicated by FGR. The number of Mops is significantly higher in women whose pregnancies resulted in term births, in contrast to women whose pregnancies resulted in preterm births [8]. It was shown that the pro-inflammatory M1 type of Mops is involved in the mechanisms of preterm birth [9]. At the same time, the role and phenotypic characteristics of M1 and M2 Mops in the pathogenetic mechanisms of pregnancy, complicated by FGR and resulting in term or preterm birth, remain completely unexplored. It is obvious that the quality and adequacy of signal transduction by macrophages can determine the course of pregnancy, its outcome and timely prognosis. Therefore, macrophages require a particular attention when considering the pathogenetic features of FGR and represent an attractive therapeutic target for the prevention of arising disorders. 


\section{THE AIM}

The aim of the current study was to elucidate the possible involvement of M1 and M2 macrophages in the placentas of women, whose pregnancies were complicated by fetal growth restriction (FGR) and resulted in term births after 37 weeks of gestation and preterm births up to 37 weeks of gestation.

\section{MATERIALS AND METHODS}

26 placentas of women whose pregnancy resulted in live birth at a gestational age from 24 to 41 weeks at the City Clinical Maternity Hospital of Poltava City Council in 2015-2017 comprised the material of the present study. Placental bed biopsies were collected from women, whose pregnancies were complicated by FGR and whose pregnancies resulted in term births after 37 weeks of gestation (1- ${ }^{\text {st }}$ group, $n=7$ ), from women, whose pregnancies were complicated by FGR and whose pregnancies resulted in preterm births within 37 weeks of gestation (2-nd group, $\mathrm{n}=9$ ) and from women with physiological pregnancies and births (control group; $n=10$ ). The study was approved by the Commission on Bioethics of Ukrainian Medical Stomatological Academy. Written consent was obtained from all study participants.

FGR was defined as birth weight less than 10th centile with an increased ratio of systolic / diastolic pressure in the umbilical artery by Doppler ultrasonography or resistance index $>$ 95th centile for gestation. All patients with FGR

Table 1. Characteristics of women with physiological pregnancies $(n=10)$, women whose pregnancies were complicated by FGR and resulted in term births $(n=7)$, and women whose pregnancies were complicated by FGR and resulted in preterm births $(n=9)$

\begin{tabular}{|c|c|c|c|}
\hline \multirow[b]{2}{*}{ Parameter } & \multicolumn{3}{|c|}{$\begin{array}{l}\text { Clinical Characteristics of Pregnancies for Placentas Studied based } \\
\qquad \text { Mean } \pm \text { SD, median }[\text { IQR] or } n(\%)\end{array}$} \\
\hline & $\begin{array}{l}\text { Women with } \\
\text { physiological } \\
\text { pregnancies } \\
n=10\end{array}$ & $\begin{array}{l}\text { Women whose pregnancies } \\
\text { were complicated by FGR } \\
\text { and resulted in term births } \\
\qquad n=7\end{array}$ & $\begin{array}{c}\text { Women whose pregnancies were } \\
\text { complicated by FGR and resulted } \\
\text { in preterm births } \\
\qquad n=9\end{array}$ \\
\hline Gravidity & $1[1-3]$ & $1[1-2]$ & $3[1-4]$ \\
\hline Parity & $1[1-2]$ & $1[1-1]$ & $1[1-2]$ \\
\hline Gestational age (weeks) & $38.9 \pm 0.99$ & $38.86 \pm 1.21$ & $34.55 \pm 1.51^{b^{* * c^{* *}}}$ \\
\hline Maternal age (years) & $27.5 \pm 5.56$ & $25.0 \pm 4.12$ & $30.33 \pm 7.48$ \\
\hline Cigarettes & $1(10)$ & $1(14.3)$ & $1(11.1)$ \\
\hline $\begin{array}{l}\text { Anesthesia: } \\
\text { Epidural } \\
\text { General }\end{array}$ & $\begin{array}{c}3(30) \\
0\end{array}$ & $\begin{array}{l}4(57.1) \\
1(14.3) \\
\end{array}$ & $\begin{array}{l}5(55.5) \\
1(11.1) \\
\end{array}$ \\
\hline $\begin{array}{c}\text { Cervical ripening agent: } \\
\text { None } \\
\text { Mechanical }\end{array}$ & $10(100)$ & $1(14.3)$ & $1(11.1)$ \\
\hline $\begin{array}{l}\text { Labor, yes } \\
\text { Labor, hours }\end{array}$ & $\begin{array}{c}8(80) \\
7.04 \pm 0.25\end{array}$ & $\begin{array}{c}5(71.4) \\
5.77 \pm 4.22 \\
\end{array}$ & $\begin{array}{c}3(33.3) \\
2.22 \pm 3.39^{b^{* *}}\end{array}$ \\
\hline $\begin{array}{l}\text { Delivery mode: } \\
\text { C-section, repeat, no labor } \\
\text { C-section, repeat, with labor } \\
\text { C-section, primary, no labor } \\
\text { C-section, primary, with labor }\end{array}$ & $\begin{array}{c}- \\
- \\
1(10) \\
1(10)\end{array}$ & $\begin{array}{c}- \\
- \\
2(28.6) \\
1(14.3)\end{array}$ & $\begin{array}{c}2(22.2) \\
- \\
3(33.3) \\
1(11.1)\end{array}$ \\
\hline $\begin{array}{c}\text { Maternal Oxygen given at } \\
\text { delivery }\end{array}$ & $2(20)$ & $3(42.9)$ & $6(66.7)$ \\
\hline Birth weight (grams) & $3321 \pm 292.5$ & $2484.3 \pm 394.8$ & $1955.6 \pm 558.9^{a^{* *} b^{* *}}$ \\
\hline Placental weight (grams) & $484.2 \pm 80.46$ & $421.1 \pm 134.8$ & $347.8 \pm 68.9^{b^{* *}}$ \\
\hline Baby's sex: Female / Male & $6(60) / 4(40)$ & $4(57.2) / 3(42.9)$ & $8(88.9) / 1(11.1)$ \\
\hline Delivery to processing (min) & $7.6 \pm 16.3$ & $16.1 \pm 20.6$ & $29.1 \pm 22.3^{b^{*}}$ \\
\hline
\end{tabular}

FGR: fetal growth restriction

a - comparisons were performed between columns with women with physiological pregnancy and the group of women whose pregnancies were complicated by FGR and resulted in term births.

$\mathrm{b}$ - comparisons were performed between columns with women with physiological pregnancy and the group of women whose pregnancies were complicated by FGR and resulted in preterm births.

c-comparisons the group of women whose pregnancies were complicated by FGR and resulted in term births with the group of women whose pregnancies were complicated by FGR and resulted in preterm births.

${ }^{*} p<0.05{ }^{* *} p<0.001$ 

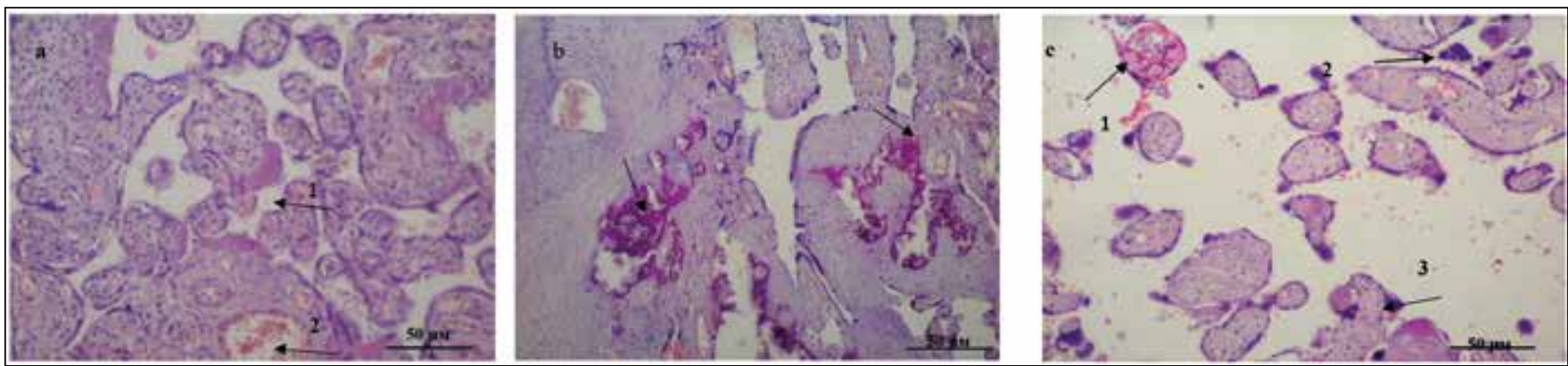

Fig. 1. (a) Terminal, intermediate and stem villi with moderate vascular blood filling, areas of fibrinoid deposition (1) in the intervillous space, general pattern of vascular congestion (2) in women with physiological pregnancies. (b) Areas of deposition of calcium salts in the stroma of the villous chorion of the placentas in the second group of women whose pregnancies were complicated by FGR and resulted in term births. Proliferation of connective tissue in the villi and obliteration of the vascular bed. (c) Avascular villi, areas of reduction of the vascular bed (1) of the placentas in women whose pregnancies were complicated by FGR and resulted in preterm births. Proliferation of syncytium with the formation of symplastic nodules (2) Areas of dystrophic calcification in the terminal villi (3). Staining with hematoxylin and eosin, magn. $\times 200$.
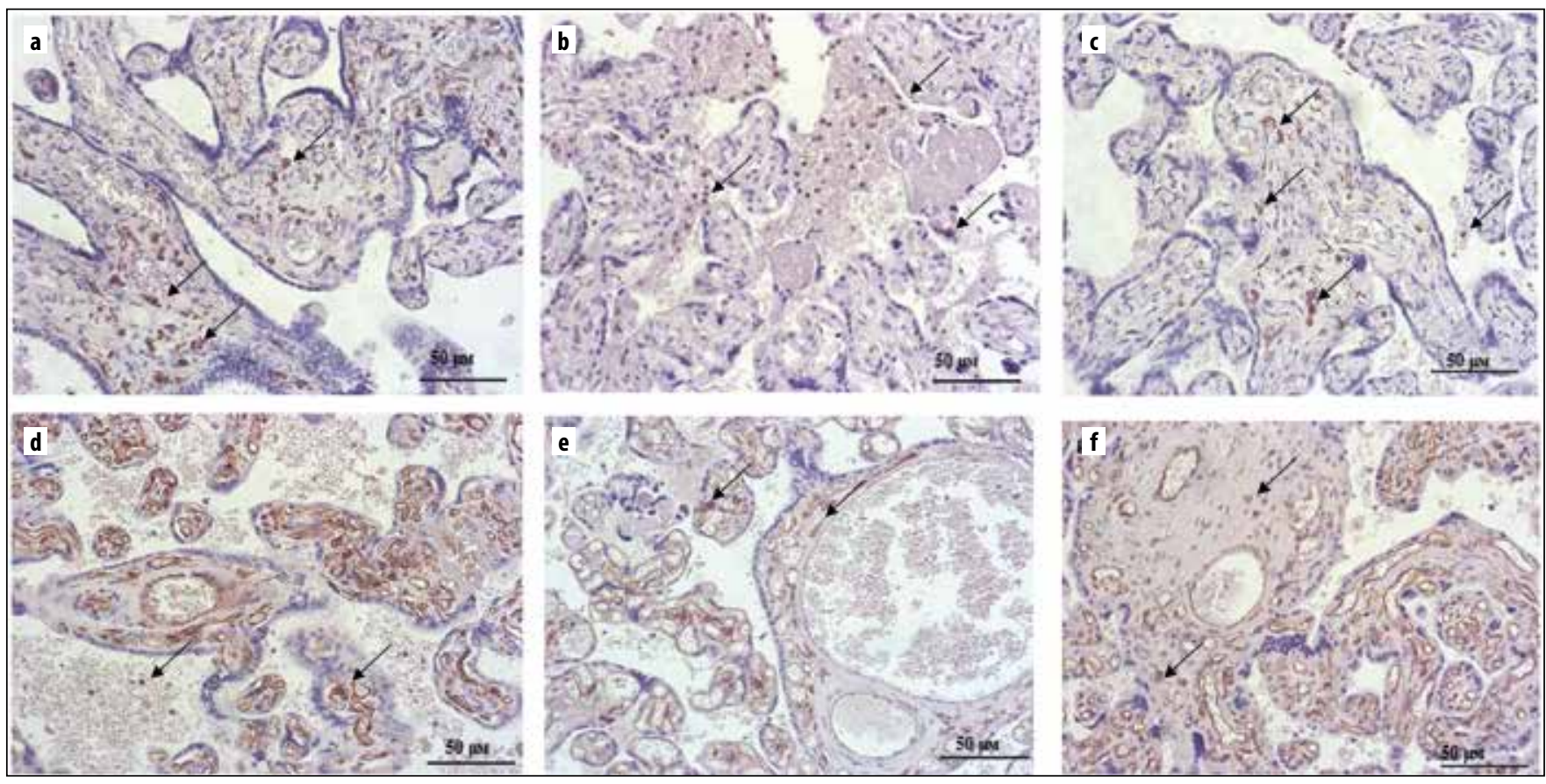

Fig. 2. (a) Expression of CD68 in women with physiological pregnancies; (b) Expression of CD68 in the placentas of women whose pregnancies were complicated by FGR and resulted in term births; (c) Expression of CD68 in the placentas of women whose pregnancies were complicated by FGR and resulted in preterm births; (d) Expression of CD163 in the placentas of women with physiological pregnancies; (e) Expression of CD163 in the placentas of women whose pregnancies were complicated by FGR and resulted in term births; (f) Expression of CD163 in the placentas of women whose pregnancies were complicated by FGR and resulted in preterm births; staining with hematoxylin, magn. x200.

underwent prenatal ultrasound examination starting from 24 weeks of pregnancy. The main criteria for inclusion in the study were as follows: pregnant women, antenatally diagnosed with FGR by ultrasound, namely, the decreased biparietal size of the fetal head, abdominal diameter, hip length and the discrepancy between these sizes and gestational age. The exclusion criteria embraced: patients with pre-existing hypertension, renal failure, diabetes mellitus, gestational diabetes.

The morphological material was fixed in $10 \%$ neutral buffered formalin, dehydrated in alcohols and embedded in paraffin. For histological verification, 6-9 pieces were excised from the organ (central, paracentral, peripheral parts of the placenta). To study the structure of the placenta, histological preparations were stained with hematoxylin-eosin. The sections were examined under microscope followed by photographing (x200, x400; Olympus BX-41, Olympus, Germany).

The expression of CD68+ and CD163+ macrophages was investigated in all samples by using immunohistochemical streptavidin peroxidase method. Paraffin sections, $4 \mu \mathrm{m}$ thick, were deparaffinized and dehydrated, antigens were recovered in citrate buffer in the microwave oven, and endogenous peroxidase was blocked. Further, the sections were incubated at $4^{\circ} \mathrm{C}$ overnight with murine monoclonal antibodies anti-CD68 (1:25, clone PG-M1, REF PD 


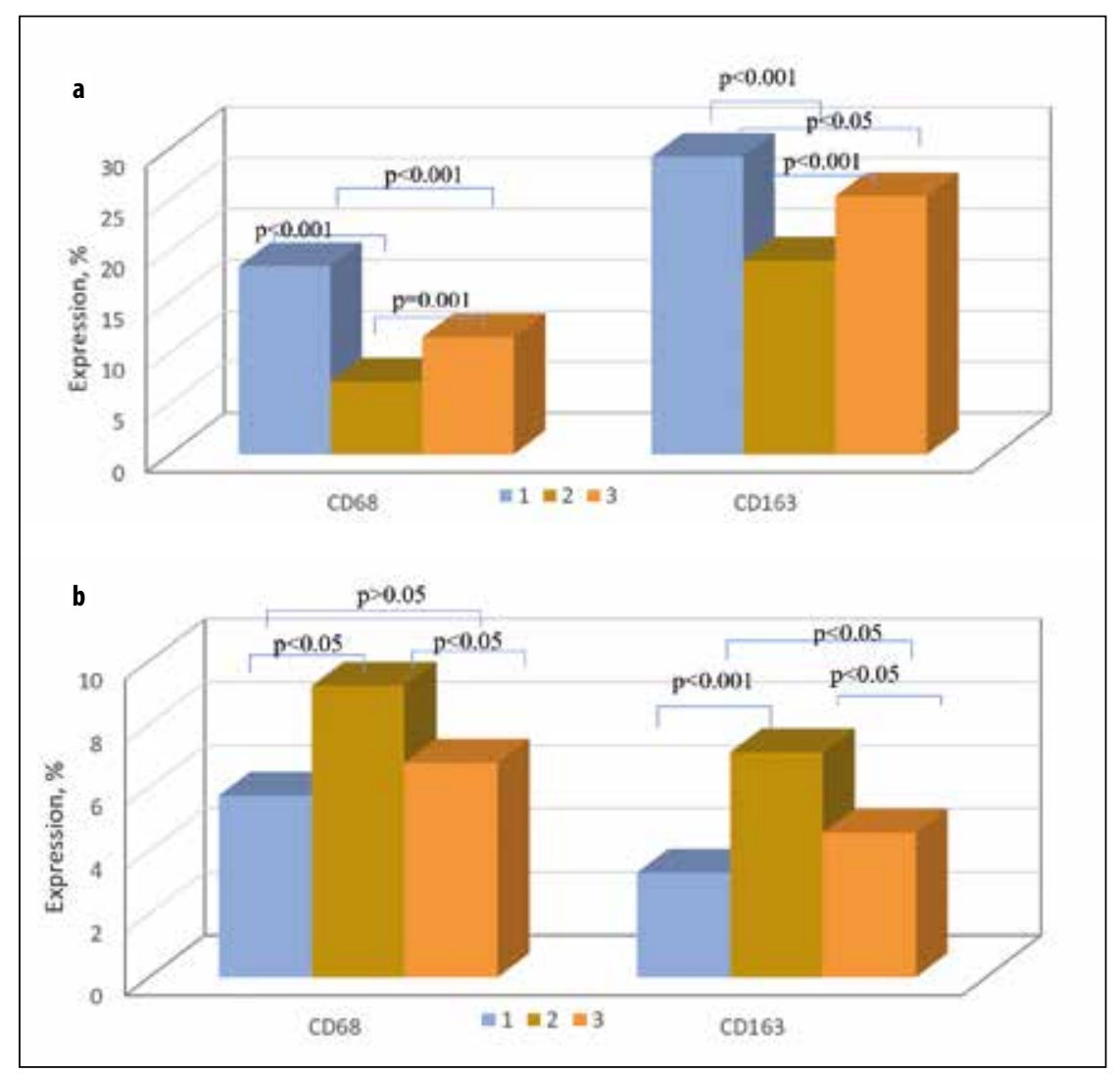

Fig. 3. (a) Indicators of the expression level of the CD68 and CD163 macrophages in the stroma of chorionic villi in women with physiological pregnancies (1), women whose pregnancies were complicated by FGR and resulted in term births (2), and women whose pregnancies were complicated by FGR and resulted in preterm births (3). (b) Indicators of the expression level of the CD68 and CD163 monocytes in the intervillous space of the chorionic villi in women with physiological pregnancies (1), women whose pregnancies were complicated by FGR and resulted in term births (2), and women whose pregnancies were complicated by FGR and resulted in preterm births (3).
M065-S, Diagnostic BioSystems, USA) and anti-CD163 ( $1: 100$, clone 10D6, REF Mob460-01, Diagnostic BioSystems, USA). Afterwards, the sections were treated in two steps with the Mouse/Rabbit PolyVue ${ }^{\mathrm{Tu}}$ HRP/DAB Detection System (Diagnostic BioSystems, USA), with visualization by chromogen; the nuclei were counterstained with Mayer's haemalaun. We used Antibody Diluent buffer as a negative control instead of primary antibodies, and lymph node tissues were used as a positive control. Quantitative indicators were obtained by counting immunopositive $\mathrm{CD}^{+} 8^{+}$and $\mathrm{CD} 163^{+}$cells over the entire field of view with a large magnification lens $\times 40$ (high power field, HPF) of placenta. We took into account all obtained quantitative individual data from all fields of view with calculating the mean value. The sections were examined under microscope followed by photographing (x200, x400; Axio Lab. A1, Zeiss, Germany)

Analyses were performed using Prism 5.0 (GraphPad, CA, USA). P-values $<0.05$ were considered to indicate statistical significance. Normally distributed data were reported using the means with standard deviations, categorical variables were reported using counts and proportions. Comparisons between groups were performed using parametric T-test and nonparametric methods: $\chi^{2}$ Fischer exact test, Spearman's correlation test.

\section{RESULTS}

The study involved women aged from 18 to 38 years (Table 1). The age of women $1-^{\text {st }}$ group ranged from 19 to 31 years. The age of women $2{ }^{\text {nd }}$ group ranged from 18 to 38 years.
The age of women in the control group - from 22 to 30 years. There were no significant differences in age between groups of women.

The analysis of the number of pregnancies and deliveries indicates that, the majority of women in all groups had their first pregnancy and delivery: among women $1-^{\text {st }}$ group 4 subjects $(57.1 \%)$ and 6 subjects (85.7\%), respectively; among women 2 - $^{\text {nd }}$ group -4 subjects (44.4\%) and 5 subjects (55.5\%), respectively; among women of the control group -7 subjects (70\%) and 7 subjects (70\%), respectively.

The gestational age at which labor developed was reliably shorter in women with FGR, whose pregnancies resulted in both1 - $^{\text {st }}$ and $2-^{\text {nd }}$ groups, in contrast to women of the control group ( $p<0.001, p<0.001$, respectively). It was found that the duration of labor was longer (by 3.8 times) in women $2{ }^{- \text {nd }}$ group, than in women of the control group $(\mathrm{p}<0.05)$.

The parameters of the body weight of newborns were significantly lower among women both 1 - $^{\text {st }}$ and $2{ }^{- \text {nd }}$ groups, than among women of the control group ( $<<0.001$, p $<0.001$, respectively). The placenta weight was lower (by 1.7 times) in women 2 -nd $^{\text {nd }}$ group, than in women of the control group $(\mathrm{p}<0.001)$.

Histological examination of the placentas of women both 1 - $^{\text {st }}$ and 2- ${ }^{\text {nd }}$ groups, in contrast to women in the control group, showed a discrepancy between its maturity and gestational age, and the disrupted maturation of chorionic villi, predominantly of the dissociative type (Fig. 1). Thus, among the mature terminal villi in the specimens of this group, were found both mature and immature intermediate villi, groups of chaotic sclerosed villi. Quite often, zones of 
involutive and dystrophic processes were registered in the form of afunctional areas, in particular, due to excessive deposition of fibrinoid in the intervillous space. In women of control group fibrinoid deposits were diffusely focal, mainly in the peripheral parts of the placenta, which indicated its disrupted maturation and inflammatory changes.

Much more often, was observed significant areas of dystrophic calcification in the placentas of women 1 -st $^{\text {stoup, }}$ in contrast to women $2{ }^{\text {nd }}$ group ( 5 subjects; $71.5 \%$ versus 1 subject, $11.11 \% ; \mathrm{p}<0.05)$. In the placentas of women $2{ }^{- \text {nd }}$ group, changes in the stroma of the villi were noted, which were characterized by an increase in the connective tissue, increased collagen formation, foci of necrosis, an increase in calcification deposits in syncytial nodules, which practically excluded their role in the adaptation process of villi.

In the placenta of women 2-nd group, more pronounced signs of impaired vascularization (hypovascularization) and hypoplasia of chorionic villi were manifested, which indicated the inability of terminal villi to carry out compensatory reactions to the full extent. In particular, the proliferation of terminal villi in the placentas of women $2{ }^{\text {nd }}$ group was much less frequently determined, in contrast to women in the control group ( 1 subject, $11.1 \%$ versus 7 subjects, $70 \% ; \mathrm{p}<0.01)$. At the same time, destructive and degenerative processes were combined with signs of compensatory and adaptive reactions in both groups of women, whose pregnancies were complicated by FGR.

In the placentas of women of the control group, were observed a sufficient level of compensatory and adaptive reactions in terminal villi with the formation of syncytial nodules, capillary hyperplasia, and active formation of syncytiocapillary membranes.

Expression of CD68 marker (Fig. 2, 3) was found in the cytoplasm of macrophages located in the stroma of intermediate and terminal villi, as well as in monocytes of the intervillous space. The level of CD68+ macrophages in terminal villi of the placenta was significantly lower in women both $1-^{\text {st }}$ and $2{ }^{\text {nd }}$ groups than in women of the control group ( $\mathrm{p}<0.001 ; \mathrm{p}<0.001$, respectively). The level of CD68+ macrophages in the placentas of women $2^{-{ }^{n d}}$ group was significantly higher than in placentas of women $1^{\text {- }}$ ts group $(p<0.001)$. The level of CD68+ monocytes in the intervillous space of the placenta was significantly higher in women $1^{- \text {st }}$ group as compared to women of the control group $(\mathrm{p}<0.05)$ and women $2{ }^{\text {nd }}$ group $(\mathrm{p}<0.05)$.

Fig. 3. (a) Indicators of the expression level of the CD68 and CD163 macrophages in the stroma of chorionic villi in women with physiological pregnancies (1), women whose pregnancies were complicated by FGR and resulted in term births (2), and women whose pregnancies were complicated by FGR and resulted in preterm births (3). (b) Indicators of the expression level of the CD68 and CD163 monocytes in the intervillous space of the chorionic villi in women with physiological pregnancies (1), women whose pregnancies were complicated by FGR and resulted in term births (2), and women whose pregnancies were complicated by FGR and resulted in preterm births (3).
Expression of CD163 marker was registered mainly on the membrane of macrophages, which were found in the stroma of terminal villi, as well as on the surface of monocytes localized in the intervillous space. The level of CD163+ macrophages in terminal villi of the placenta was significantly lower in women both $1-^{\text {st }}$ and $2{ }^{- \text {nd }}$ groups, in contrast to women of the control group ( $p<0.001$, $\mathrm{p}<0.001$, respectively). The level of CD163+ macrophages in this compartment of the placenta was significantly lower in women $1^{- \text {st }}$ group as compared to women $2{ }^{\text {nd }}$ group $(\mathrm{p}<0.001)$. The level of CD163+ monocytes in the intervillous space of the placenta is significantly higher in women $1-^{\text {st }}$ group as compared to women of the control group $(\mathrm{p}<0.001)$ and women $2{ }^{\text {nd }}$ group $(\mathrm{p}<0.05)$. A significantly higher level of CD163+ monocytes in the intervillous space was revealed in women 2 -nd group as compared to women in the control group $(\mathrm{p}<0.05)$.

Analysis of the expression ratio of CD68+ / CD163+ macrophages subpopulations demonstrated that in the stroma of terminal villi the $\mathrm{M} 2$ profile significantly predominates over M1 in the placentas of women of the control group ( $\mathrm{p}$ $<0.001)$, as well as in women $1^{\text {- }}{ }^{\text {st }}$ group $(\mathrm{p}<0.001)$ or $2^{\text {-nd }}$ group $(\mathrm{p}<0.001)$. Analysis of the expression ratio of CD68+ / CD163+ monocytes subpopulations demonstrated that in the intervillous space, the M1 pro-inflammatory profile exceeds the M2 anti-inflammatory profile in the placentas of women of the control group ( $\mathrm{p}<0.05)$, as well as women 2 - $^{\text {nd }}$ group $(\mathrm{p}<0.001)$.

In women of the control group, correlation analysis revealed a significant relationship between the expression levels of CD68+ and CD163+ macrophages in the stroma of terminal villi of the placenta $(r=-0.787 ; p=0.007)$, and in women $2{ }^{\text {nd }}$ group - between the levels of CD68+ monocytes in the intervillous space and the weight of a newborn $(r=-0.765 ; p=0.016)$.

\section{DISCUSSION}

In the current study it was shown that in women $2{ }^{-{ }^{\text {nd }}}$ group was lower indicators of the placenta weight; they gave birth at an earlier gestational age and after a prolonged labor period, in contrast to women 1 - $^{\text {st }}$ group or women control group. Our findings are consistent with studies that investigated the relationship between the postpartum placental morphometry and infant birth weight. It has been shown that low birth weight is comparable to low parameters of weight, volume and area of the placenta in FGR [10]. As our results have shown, in FGR, there are characteristic signs of placental insufficiency. Vascularization, development and growth of villi are particularly important morphological indicators of the state of the placenta. Meanwhile, in the placentas of women $1^{- \text {st }}$ group, we identified the morphological changes that indicate the presence of a state of hyperfunction with inclusion of compensatory and adaptive mechanisms. At the same time, the changes in the placentas of women 2-nd group indicate the insufficiency of compensatory processes, manifested by the disrupted vascular and stromal component of the villous chorion and the development of involutive and 
dystrophic changes. It has been suggested that morphological changes in the placenta in FGR may be caused by placental perfusion due to a decrease in the vascular bed and hypovascularization of terminal villi [11].

Macrophages (Hofbauer cells) play a crucial role in pregnancy, and their dysfunction or alteration of polarity is involved in pregnancy disorders, like FGR and preterm labor. We have demonstrated that macrophages with CD68+ and CD163+ phenotypes are detected in all groups of placentas. It is likely that macrophages are influenced by factors of the local microenvironment, which contributes to polarization, taking into account the conditions in the placenta during pregnancy. However, one cannot exclude the influence of macrophages themselves on a number of processes in the placenta, including their control over the development and remodeling of chorionic villi; control over angiogenesis of villi; control of trophoblast transformation and the formation of syncytial nodules [12].

Our data on the prevalence of CD163+ M2 macrophages population over CD68+ phenotype of M1 macrophages in the stroma of terminal villi of the placenta in all groups of women are partially consistent with other studies, which assume that CD163+ macrophages (Hofbauer cells) are constitutively expressed in all compartments of the placenta throughout pregnancy, whereas CD68+ macrophages have variable expression over time, and its intensity peaks in the second trimester and decreases as pregnancy progresses $[13,14]$. At the same time, it is important to note that the functions of M1 and M2 macrophages in the placenta in FGR remain completely unclear and contradictory.

It was found a more pronounced increase in the number of CD68+ macrophages in the placenta of women 2-nd group, in contrast to the indicators of women $11^{\text {st }}$ group, and these findings are consistent with the results of other authors. It is likely that the increased level of the pro-inflammatory profile of CD68+ macrophages in preterm labor can be explained by the participation of these cells in several processes (maturation of the fetoplacental tissue, preparation of the uterus for childbirth, etc.), which are accompanied by activation of inflammatory processes [15]. The revealed overexpression of CD68+ pro-inflammatory monocytes in women whose pregnancies resulted in preterm births is consistent with the findings of several authors [16], indicating that peripheral blood mononuclear cells entering the intervillous space can begin to differentiate into mature M1 macrophages under the influence of M1-activating inflammatory environmental stimuli. However, if necessary, they can repolarize to M0 or even M2 macrophages, depending on the needs and influence of the microenvironmental factors [17].

\section{CONCLUSIONS}

Thus, in FGR, the placenta undergoes the development of pathomorphological changes characteristic of placental insufficiency. In the present study, in the placentas of women whose pregnancies were complicated by FGR and resulted in term births underwent transformation of the chorionic villi and the vascular component, a decrease in the number of CD68+ M1 profile macrophages with a predominance of CD163+ M2 macrophages population of the placenta as opposed to physiological pregnancies. Therefore, one can observe the disruptions of compensatory mechanisms with their partial adaptation, since CD68+ macrophages of the pro-inflammatory pool are directly associated with inflammatory infiltration of the placenta and an increase in the proliferation of terminal villi with compensation of metabolic processes, whereas CD163+ macrophages of the anti-inflammatory pool affect the formation of hemodynamic adaptive reactions.

Finally, in the placentas of women whose pregnancies were complicated by FGR and resulted in preterm births, there were disruptions of the vascular and stromal component of the chorionic villus with the development of involutive and dystrophic changes due to a decrease in the average area of terminal villi. In this case, the role of macrophages should be considered in a much broader aspect. In our opinion, their participation is associated with a disruption of compensatory mechanisms in the placenta, since the number of the CD68+ macrophages of the pro-inflammatory pool is negatively associated with inflammatory infiltration of the placenta. This is presumably due to dysregulatory processes caused by hypovascularization that is also associated with a reduced birth weight, which generally may determine the development of chronic placental insufficiency and enhance the formation of FGR. This results will contribute to improve the prognosis and diagnosis of FGR and fetal prematurity.

\section{REFERENCES}

1. Vijayaselvi R., Cherian A. Risk assessment of intrauterine growth restriction. Curr. Med. Issues. 2017;15(4):262. https://doi:10.4103/cmi. cmi_76_17.

2. Hromova A.M., Berezhna V.A. Akushers'ki y antenatal'ni faktory ryzyku zatrymky vnutrishn'outrobnoho rostu ploda [Obstetric and antenatal risk factors for intrauterine growth restriction]. Zaporozhye Med. Journal. 2020; 22 (3):395-401. (In Ukrainian). https://doi.org/10.14739/23101210.2020.3.204949.

3. Benton S.J., Mc Cowan L.M., Heazell A.E.P. et al. Placental growth factor as a marker of fetal growth restriction caused by placental dysfunction. Placenta. 2016;42:1-8. https://doi.org/10.1016/j. placenta.2016.03.010.

4. Lee K.Y. M1 and M2 polarization of macrophages: a mini-review. Med. Biol. Sci. Eng. 2019; 2 (1): 1-5. https://doi.org/10.30579/ mbse.2019.2.1.1

5. Kaidashev I.P., Yemchenko Ya.0., Shynkevych V.I. et al. PPAR-Gamma Agonist Pioglitazone Reduced CD68+ but Not CD163+ Macrophage Dermal Infiltration in Obese Psoriatic Patients. PPAR Res. 2020; 2020: 4548012. https://doi.org/10.1155/2020/4548012.

6. Bezemer R.E., Schoots M.H., Timmer A. et al. Altered levels of decidual immune cell subsets in fetal growth restriction, stillbirth, and placental pathology. Front. Immunol. 2020; 11:1-14. https://doi.org/10.3389/ fimmu.2020.01898.

7. Svensson-Arvelund J., Mehta R.B., Lindau R. et al. The human fetal placenta promotes tolerance against the semiallogeneic fetus by inducing regulatory $\mathrm{T}$ cells and homeostatic M2 macrophages. J. Immunol. 2015;194 (4): 1534-1544. https://doi: 10.4049/ jimmunol.1401536. 
8. Gomez-Lopez N., Vega-Sanchez R., Castillo-Castrejon M. et al. Evidence for a role for the adaptive immune response in human term parturition. Am. J. Reprod. Immunol. 2013; 69 (3):212-230. https://doi: 10.1111/ aji.12074.

9. Gonzalez J.M., Franzke C.W., Yang F. et al. Complement activation triggers metalloproteinases release inducing cervical remodeling and preterm birth in mice. Am. J. Pathol. 2011; 179 (2): 838-849. https:// doi: 10.1016/j.ajpath.2011.04.024.

10. Balihallimath R.L., Shirol V.S., Gan A.M. et al. Placental morphometry determines the birth weight. J. Clin. Diagnostic. Res. 2013; 7(11): 2428-2431. https://doi: 10.7860/JCDR/2013/7478.3564.

11. Junaid T.0., Brownbill P., Chalmers N. et al. Fetoplacental vascular alterations associated with fetal growth restriction. Placenta. 2014; 35(10): 808-815. https://doi:10.1016/j.placenta.2014.07.013.

12. Graham C., Chooniedass R., Stefura W.P et al. In vivo immune signatures of healthy human pregnancy: Inherently inflammatory or antiinflammatory? PLoS One. 2017; 12 (6): 1-16. https://doi: 10.1371/ journal.pone.0177813.

13. Ingman K., Cookson V.J.K.W., Jones C.J.P. et al. Characterisation of Hofbauer cells in first and second trimester placenta: incidence, phenotype, survival in vitro and motility. Placenta. 2010; 31(6): 535-544. https://doi:10.1016/j.placenta.2010.03.003.

14. Reyes L., GolosT.G. Hofbauer cells: Their role in healthy and complicated pregnancy. Front. Immunol. 2018;9: 1-8. https://doi: 10.3389/ fimmu.2018.02628.

15. Gomez-Lopez N., StLouis D., Lehr M.A. et al. Immune cells in term and preterm labor. Cell Mol. Immunol. 2014;11 (6):571-581. https://doi: 10.1038/cmi.2014.46.

16. Das A., Ganesh K., Khanna S. et al. Engulfment of apoptotic cells by macrophages: a role of micro-RNA-21 in the resolution of wound inflammation. J. Immunol. 2014; 192 (3): 1120-1129. https:// doi:10.4049/jimmunol.1300613.

17. Schliefsteiner C., Ibesich S., Wadsack C. Placental Hofbauer cell polarization resists inflammatory cues in vitro. Int. J. Mol. Sci. 2020; 21 (3): 736. https://doi:10.3390/ijms21030736.
The present article is a part of the research project of Ukrainian Medical Stomatological Academy "The study of the pathogenetic role of the circadian molecular clock in the development of metabolic diseases and systemic inflammation and development of treatment methods aimed at the processes”. Completion period: 2020-2022 (№ state registration 0120U101166).

\section{ORCID and contributionship:}

Varvara A. Berezhna: 0000-0002-9251-8100 A,B,C,D,E,F

Tetiana V. Mamontova: 0000-0003-4967-9379 ${ }^{B, C, F}$

Antonina M. Gromova: 0000-0002-7396-7023 $3^{A, E, F}$

\section{Conflict of interest:}

The Authors declare no conflict of interest.

\section{CORRESPONDING AUTHOR Varvara A. Berezhna \\ Ukrainian Medical Stomatological Academy \\ 27B 0. Honchara St., 36039 Poltava, Ukraine \\ tel: +380504048411 \\ e-mail: berejnayapoltava@gmail.com}

Received: 09.07.2020

Accepted: 04.01.2021

A - Work concept and design, B - Data collection and analysis, C - Responsibility for statistical analysis, D-Writing the article, $\mathbf{E}$-Critical review, $\mathbf{F}$ - Final approval of the article 